\section{Investigating graphene oxide permeable reactive barriers for filtering groundwater contaminated from hydraulic fracturing}

\author{
Zifeng An, Konrad Grala, Aakanx Panchal and Kunjan Trivedi
}

\begin{abstract}
Hydraulic fracturing, or fracking, is a method of natural gas extraction which involves pumping a brine solution into the ground to create a fracture that will allow natural gas to rise. One of the major concerns surrounding this method of natural gas extraction is that wastewater enters the groundwater supply, thereby contaminating it. This wastewater contains toxic materials such as heavy metal ions, radionuclides and other salts and organic compounds in high concentrations. Some of these materials are carcinogenic and thus a concern to human life and the environment. The current solution involves the use of a zerovalent iron (ZVI) permeable reactive barrier (PRB) to filter out these toxic substances. However, it causes more fouling due to the accumulation of mineral precipitates and therefore is not very effective. A recent development in nanotechnology may allow us to develop a superior water filter to prevent groundwater contamination. Therefore, a novel PRB is suggested: featuring the use of solid graphene oxide (GO), a nanomaterial with a superior sorption ability is proposed as a replacement for the system. The proposed experiment will test the filtration capability of the GO-PRB as compared to the traditional ZVI-PRB. By emulating the process of groundwater contamination and flow using common materials found in fracking wastewater, we can determine how much more effective the GO-PRB is than the ZVIPRB.
\end{abstract}

\section{Résumé}

La fracturation hydraulique est une méthode d'extraction de gaz naturel qui consiste à pomper une solution de saumure dans le sol pour créer une fracture qui permettra au gaz naturel de monter. L'une des principales préoccupations entourant cette méthode d'extraction du gaz naturel est que les eaux usées pénètrent dans l'eau souterraine et la contaminent. Cette eau usée contient des matières toxiques telles que des ions de métaux lourds, des radionucléides et d'autres sels et composés organiques à des concentrations élevées. Certains de ces matériaux sont cancérigènes et donc préoccupants pour la vie humaine et l'environnement. La solution actuelle implique l'utilisation d'une barrière réactive perméable (BRP) en fer zéro valent (FZV) pour filtrer ces substances toxiques. Cependant, elle n'est pas très efficace puisqu'elle provoque plus d'encrassement dû à l'accumulation de précipités minéraux. Un développement récent en nanotechnologie peut nous permettre de développer un filtre à eau supérieur pour empêcher la contamination des eaux souterraines. Par conséquent, une nouvelle BRP est proposée qui utilise l'oxyde de graphène solide $(\mathrm{OG})$, un nanomatériau doté d'une capacité de sorption supérieure est proposé comme remplacement pour le système. L'expérience proposée testera la capacité de filtration de la GO-BRP par rapport à la FZV-BRP traditionnelle. En imitant le processus de contamination et d'écoulement des eaux souterraines à l'aide de matériaux communs trouvés dans les eaux usées de fracturation, nous pouvons déterminer à quel point la GO-BRP est plus efficace que la FZV-BRP.

\footnotetext{
*McMaster University, Hamilton, Ontario, Canada

Université McMaster, Hamilton, Ontario, Canada

${ }^{\dagger}$ Lower division / Division inférieure

$\ddagger$ 1st place / 1ère place
} 